but the synthesizing filters are fairly complicated because they contain many integrating circuits that require resetting.

\section{ACKNOWLEDGMENT}

The author would like to thank Professor H. Nishihara for his support of this work and reading and useful sug- gestion of this article, and $\mathrm{Y}$. Shimidzu for the construction of this prototype correlator.

${ }^{1}$ F. F. Chen, Sci. Amer. 217, 76 (July 1967).

${ }^{2}$ S. Ichimaru, J. Phys. Soc. Japan 19, 1207 (1964).

3 P. J. Kindlmann and E. B. Hooper, Jr. Rev. Sci. Instrum. 39 864 (1968)

${ }^{4}$ D. G. Lampard, Proc. IEE, Pt. C 102, 35 (March 1955).

\title{
A High Sensitivity Bridge Detector Circuit System for Microwave Resonance Measurement
}

\author{
I. TAKAO AND K. WATANABE \\ Research Institute of Electronics, Shizuoka University, Hamamatsu, 430, Japan
}

(Received 29 December 1970; and in final form, 8 February 1971)

\begin{abstract}
A high sensitivity detector system for microwave resonance measurements utilizing a bridge balancing method is described. This system consists of a parametron preamplifier, a phase modulated pump circuit, and a phase discriminator. The preamplifier amplifies the measuring signal and the quadrature AFC error signal alternatively.
\end{abstract}

$\mathrm{M}^{\mathrm{I}}$ ICROWAVE resonance measuring systems which utilize the bridge balancing method, such as electron spin resonance (ESR) spectrometer and microwave biased photoconductor detector, ${ }^{1}$ usually require high sensitivity detection of two distinct signals : the measuring signal and the AFC error signal. The measuring signal arises from the ESR absorption or photoconductive effect of material inserted in a microwave resonant cavity. The AFC error signal, which is in quadrature with the measuring signal, arises from the frequency fluctuations of the klystron and is indispensable for high sensitivity operation.

This paper presents a microwave circuit system which permits high sensitivity detection of the two distinct signals with only one low noise, phase sensitive parametron amplifier ${ }^{2}$ in conjunction with a phase modulated pumping source.

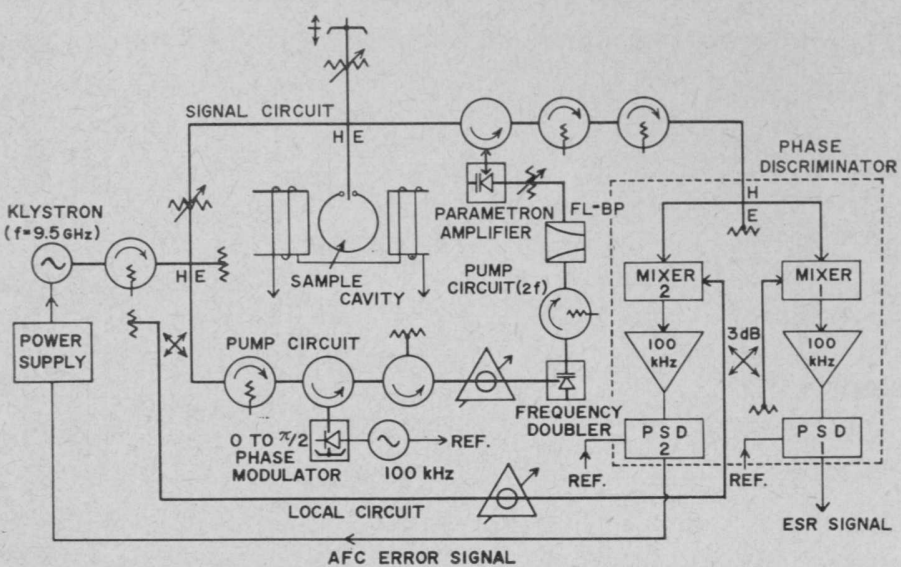

Fig. 1. The high sensitive microwave bridge detector circuit employed in a parametron ESR spectrometer with phase modulated pumping source; PSD - phase sensitive detector.
A block diagram of an X-band parametron ESR spectrometer based on this principle is shown in Fig. 1. The klystron supplies the microwave power to the signal circuit and also to the pump circuit through the varactor frequency doubler. Referring to Fig. 1, the input signal of the parametron amplifier can be written as

$$
a(t)=A \cos \left(\omega t+\theta_{a}\right),
$$

and the pumping voltage as

$$
e(t)=E \cos \left(2 \omega t+\theta_{p}\right),
$$

where it is relevant that the pump frequency is twice that of the input signal frequency. In this case, it is possible to show $^{2}$ that the gain of the parametron amplifier becomes a maximum when

$$
\theta_{p}=2 \theta_{a}+\pi / 2
$$

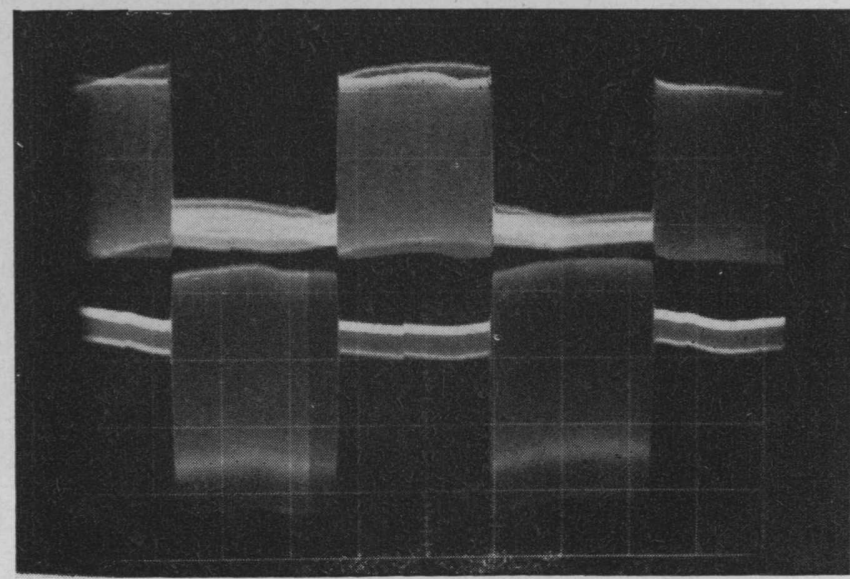

Fig. 2. Output signals of the two phase sensitive detectors of Fig. 1. The input signal was phase modulated by $90^{\circ}$ at $100 \mathrm{~Hz}$ repetition rate. 
or

$$
2 \theta_{a}-3 \pi / 2 \text {, }
$$

and becomes a minimum when

$$
\theta_{p}=2 \theta_{a}-\pi / 2
$$

or

$$
2 \theta_{a}+3 \pi / 2 \text {. }
$$

Also, when the input signal is in quadrature with that given by Eq. (1), the phase relation [Eq. (3)] gives the minimum gain and Eq. (4) the maximum gain. Therefore, it is possible to amplify alternatively two distinct signals which are $90^{\circ}$ out of phase wlth each other by switching the phase of the pump voltage by $180^{\circ}$. This is achieved in the circuit shown in Fig. 1 by the $90^{\circ}$ phase modulator provided in the pump circuit. It consists of a circulator and a $\mathrm{p}-\mathrm{i}-\mathrm{n}$ diode mounted $\lambda_{g} / 8$ away from the short in an X-band waveguide. The two time divided output signals of the parametron amplifier are then fed into the phase discriminator which consists of a magic $\mathrm{T}$ and two homodyne detectors as shown in the figure, where they are separated.
Preliminary experiments were performed using a phase shifter in the signal circuit instead of the bridge balancing circuit shown in Fig. 1. The $\mathrm{p}-\mathrm{i}-\mathrm{n}$ diode used in the phase modulator was an HPA-3001 and was driven by a rectangular voltage waveform at $100 \mathrm{kHz}$. Figure 2 is an oscillogram of the output signals of the two $100 \mathrm{kHz}$ amplifiers shown in Fig. 1. In this measurement, the phase of the input signal of the parametron amplifier was switched by $90^{\circ}$ by the use of a varactor phase modulator operating at $100 \mathrm{~Hz}$. The input power level was about $-70 \mathrm{dBm}$. It is seen, as expected, that the two signals are separated and amplified alternatively.

The authors are much indebted to T. Shirai for his help in the experiments. Thanks are also due to our colleague Dr. S. Mizushina for improvlng this manuscript.

${ }^{1}$ H. S. Sommers, Jr., Semiconductor and Semimetals, edited by R. K. Willardson and A. C. Beer (Academic, New York, 1970), Vol. 5, pp. 435-465.

2 I. Takao and T. Hayashi, IEEE Trans. Microwave Theory. Tech. MTT-17, 107 (1969).

\title{
A Cavity Search Spectrometer for Free Radical Microwave Rotational Absorption Studies*
}

\author{
Lawrence W. Hrubesh, $\dagger$ Roger E. Anderson, and Edgar A. Rinehart $\ddagger$ \\ Lawrence Radiation Laboratory, University of California, Livermore, California 94550
}

(Received 10 September 1970; and in final form, 21 January 1971)

\begin{abstract}
A Zeeman modulated microwave spectrometer, specifically designed for $\mathrm{K}$ band rotational studies of gaseous free radicals and transient molecular species and using a Fabry-Perot type resonator as the absorption cell, is described. The theoretical sensitivity of the system is shown to be comparable to that of the most sensitive waveguide cell spectrometers. The previously assigned spectra of $\mathrm{OH}$ and $\mathrm{NO}_{2}$ radicals have been successfully located with the system. The utility of the system as a search spectrometer for free radicals has been substantiated by locating previously unreported spectra for the $\mathrm{NO}_{2}$ molecule and $\mathrm{NF}_{2}$ (difluoroamino) free radical.
\end{abstract}

\section{INTRODUCTION}

$P$ AST studies of microwave rotational spectra of free radicals and other transient molecular species have suggested the need for a very sensitive, broad band sweepable system capable of searching for unknown spectra. Some ordinary waveguide cell spectrometers have both the desired sensitivity and sweep capabilities, but generally these are not useful for studies of highly reactive and potentially corrosive molecular species. Also, waveguide cell spectrometers are not easily adapted to the magnetic modulation schemes that are most appropriate for studying the paramagnetic free radicals.

An effective free radical spectrometer requires special design criteria. For example, some means must be incorporated for producing the short lived radical species very near to or, if possible, in the microwave absorption cell. Metal surfaces in the absorption cell must be eliminated or reduced to a minimum because of their affinity for the unpaired electrons of the free radical molecules. Electronic considerations include extreme demands for both long and short term frequency stability, as well as the ability to sweep search for unknown spectra. Magnetic (Zeeman) modulation techniques are needed to give high sensitivity with phase sensitive detection as well as to discriminate between paramagnetic and diamagnetic molecules. Most important, however, is the need for the system to have optimum sensitivity for detecting minute concentrations of transient species.

To date, several spectrometer systems specifically designed for free-radical or transient molecules have been 
reported..$^{1-4}$ All of these are limited in their ability to sweep a large frequency region continuously for unknown spectral lines. In this paper we report a microwave system capable of continuous search, which incorporates all the design objectives for an effective free radical spectrometer and uses a Fabry-Perot reflection type resonator as the absorption cell.

\section{RESONANT CAVITY ABSORPTION CELL}

A high $Q$ resonant cavity is ideally suited as the absorption cell of a search spectrometer. The effective absorption path length of a resonating cavity is proportional to its quality factor $(Q)$ and can therefore be made very large. This, coupled with the large volume-to-surface ratio in an open Fabry-Perot type structure, gives such a cell a distinct advantage over the ordinary waveguide cell.

The effect of saturation is often a limiting factor on the use of cavities as absorption cells for high resolution spectrometers. In a search spectrometer, however, this is not of as great concern; one can operate at higher pressures to avoid saturation. Work by Harrington ${ }^{5}$ has shown that the maximum microwave absorption signal increases linearly with molecular concentration (and therefore with pressure). Operating pressures that are too high, however, tend to decrease the sensitivity because the excessive line broadening causes inefficient Zeeman modulation for the weak modulating fields used. The only evidence of power saturation in this high $Q$ cavity spectrometer was on the $\mathrm{NH}_{3}(3,3)$ inversion line for cell pressures below $30 \mathrm{mT}$ Torr and input power levels of $5 \mathrm{~mW}$. All subsequent experiments have been carried out at pressures between 100 and $300 \mathrm{~m}$ Torr and input power levels of $10 \mathrm{~mW}$. No saturation effects have been observed at these operating conditions.

The theory of the response of a gas filled resonator has been considered ${ }^{6}$ and suggests that the maximum change in the voltage reflected from a single ended cavity when the gas absorption is coincident with the cavity resonance can be given by

$$
(\Delta V)_{\max }=\frac{Q_{L} V_{0}}{\Delta \nu} \frac{4 \pi}{h}\left|\mu_{i j}\right|^{2}\left(N_{i}-N_{j}\right),
$$

where $Q_{L}$ is the loaded $\mathrm{Q}$ of the cavity, $V_{0}$ is the voltage amplitude of the wave incident on the resonator coupling port, $\mu_{i j}$ is the matrix element of the dipole moment of the molecule for the transition involved, $N_{j}$ is the number of molecules in the higher energy state of the two states $i$ and $j, N_{i}$ is the number of molecules in the lower energy state of the two states $i$ and $j, \Delta \nu$ is $1 / 2 \pi \tau$, where $\tau$ is the mean time between collisions for the molecule involved in the absorption, and $h$ is Planck's constant.

The minimum detectable absorption can be derived from Eq. (1) and thermal noise considerations as

$$
(\gamma)_{\min }=\left(\frac{4 k T N \Delta f}{P_{0}}\right)^{\frac{1}{2}}\left(\frac{2 \pi}{Q_{L} \lambda}\right),
$$

where $P_{0}=V_{0}^{2} / Z_{0}=$ incident microwave power, $k$ is Boltmann's constant, $T$ is absolute temperature, $N$ is the noise figure of the detection system, $\Delta f$ is the bandwidth of the receiver, and $Z_{0}$ is the characteristic impedance of the connecting waveguide.

This equation suggests that maximum sensitivity from the cavity spectrometer is possible by maximizing the loaded $Q$ and input power, and by operating with small receiver bandwidths.

A numerical example of nearly optimum parameters indicates the possible sensitivity. When $T=293 \mathrm{~K}$, $\lambda=1.25 \mathrm{~cm}, Q_{L}=3 \times 10^{4}, N=100, \Delta f=1 \mathrm{~Hz}$, and $P_{0}$ $=10^{-2} \mathrm{~W}$,

$$
(\gamma)_{\min }=0.23 \times 10^{-11} \mathrm{~cm}^{-1},
$$

where $(\gamma)_{\min }$ is the coefficient of absorption for the gas sample. This value would be near the experimental optimum in detection of weak absorption with the cavity spectrometer (without signal averaging techniques).

Unfortunately, since there exists no known absolute line intensity information for any of the paramagnetic species investigated thus far, there has been no way to determine, by comparison, the actual experimental sensitivity of the Zeeman modulated system.

\section{FREQUENCY TUNING AND STABILIZATION}

The cavity itself offers a means of both stabilizing and sweeping the frequency of a voltage tunable microwave (backward wave) oscillator (BWO). The sharp absorption of microwave power near a resonant frequency of a reflection type cavity is accompanied by a phase shift in the carrier frequency and its sidebands (if it is modulated). Close to the resonant frequency, the phase changes quickly (depending on how high the $Q$ value is) from a positive phase angle to a negative one, passing through zero phase angle at exactly the resonant frequency of the cavity. It is then electronically possible to derive a dc error signal proportional to the phase change and apply this signal to tune a control electrode in the voltage tunable microwave source. The direction of the error signal can then be such as to change the frequency of the oscillator to keep the phase change to a minimum, thus stabilizing the frequency of the oscillator to the resonance peak of the cavity response.

Now any change in the physical dimensions of the cavity will change its resonating frequency. Hence the ultimate frequency stability of an oscillator that is locked to the cavity response will be limited by the mechanical movement of the cavity plates. Barring any mechanical vibra- 


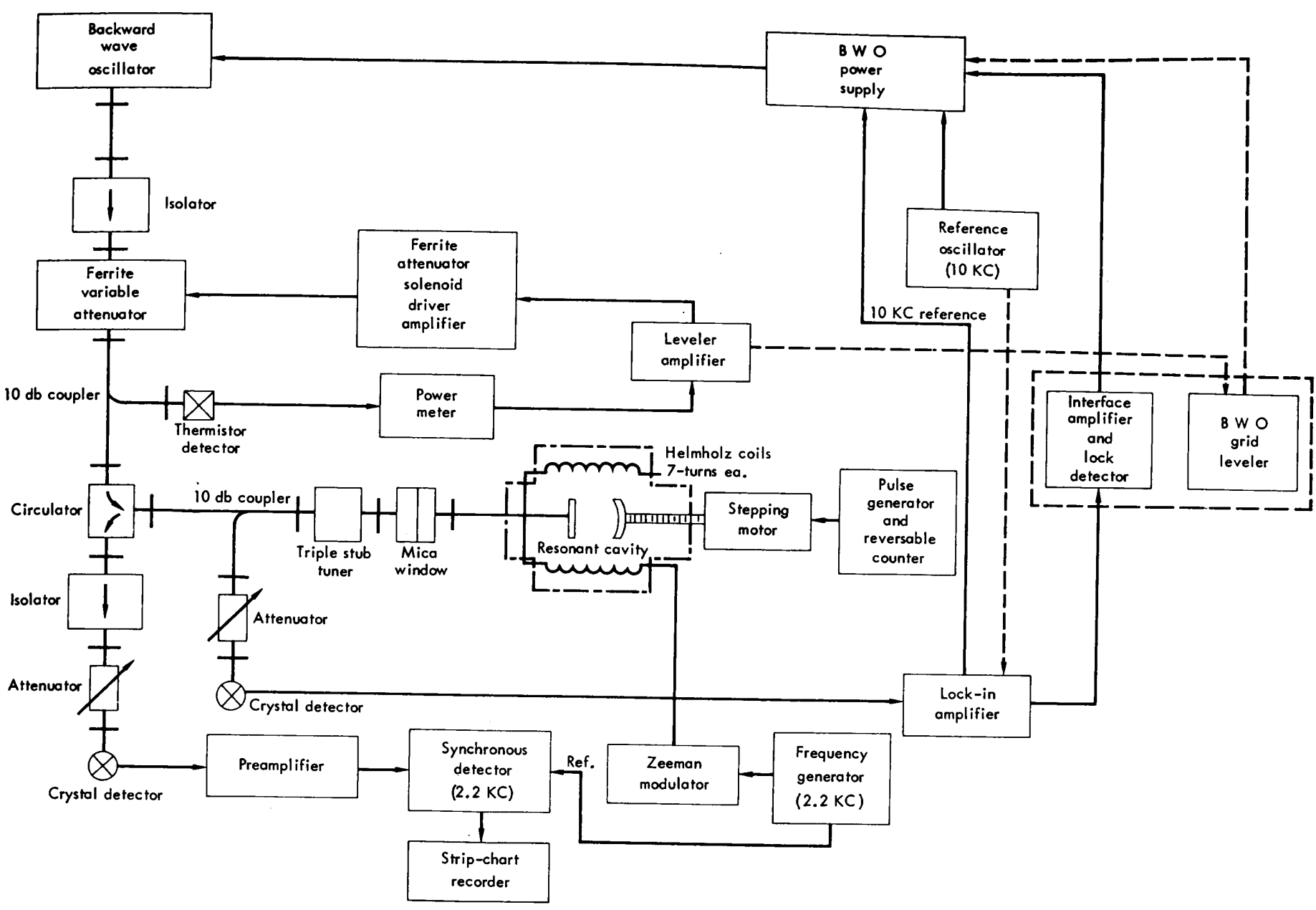

FIG. 1. Microwave resonator spectrometer.

tions of the plates, the change in cavity dimensions is usually slow and depends on environmental temperature changes. However, the spectrometer described in this paper makes use of the fact that the oscillator tracks the cavity resonance to provide a sweepable frequency. Thus it is possible to change the frequency at any rate by simply changing the rate of change of the plate separation of the resonator with a variable speed stepping motor. This technique is especially useful where the cavity doubles as the absorption cell of the spectrometer because the oscillator frequency is always locked to the peak of the cavity resonance, where it is shown in the last section to have the highest sensitivity for the detection of a simultaneous absorption by gas molecules in the cell.

The resonant frequencies for a semiconfocal FabryPerot type resonator can be shown to be given ${ }^{7}$ by

$\nu=\frac{c}{8 d}[2 q+(m+n+1)$

$$
\left.\times\left(1-\frac{2}{\pi} \tan ^{-1}\left\{\frac{2 d-b}{2[d(b-d)]^{3}}\right\}\right)\right],
$$

where $c$ is speed of light, $d$ is the separation between the plates, and $b$ is the radius of curvature of the curved plate, and $q, m$, and $n$ are integers which determine the $\mathrm{TEM}_{m n q}$ resonant mode for the cavity. It can be shown from Eq. (4) that the resonant modes of this type of resonator are sufficiently separated in frequency that one mode can be tuned through several octaves of frequency range before it becomes degenerate with another mode. This effectively makes possible the design of a very broad banded, frequency swept, microwave spectrometer system. By making the appropriate phase adjustments in an automatic frequency control (AFC) loop, the spectrometer system is able to sweep the entire frequency range of the voltage tunable oscillator continuously while keeping the oscillator stabilized to the resonant peak of the cavity.

As shown in Fig. 1, the automatic frequency control for the system is facilitated by the following electronics. A $10 \mathrm{kHz}$ reference oscillator in the lock-in amplifier modulates the microwave carrier frequency from the BWO through an amplifier in the BWO power supply. The frequency modulated carrier enters the reflection type cavity through a waveguide circulator. The reflected microwave carrier and its modulation sidebands are phase shifted in proportion to the frequency difference between the carrier and the cavity resonant frequency. The detector 


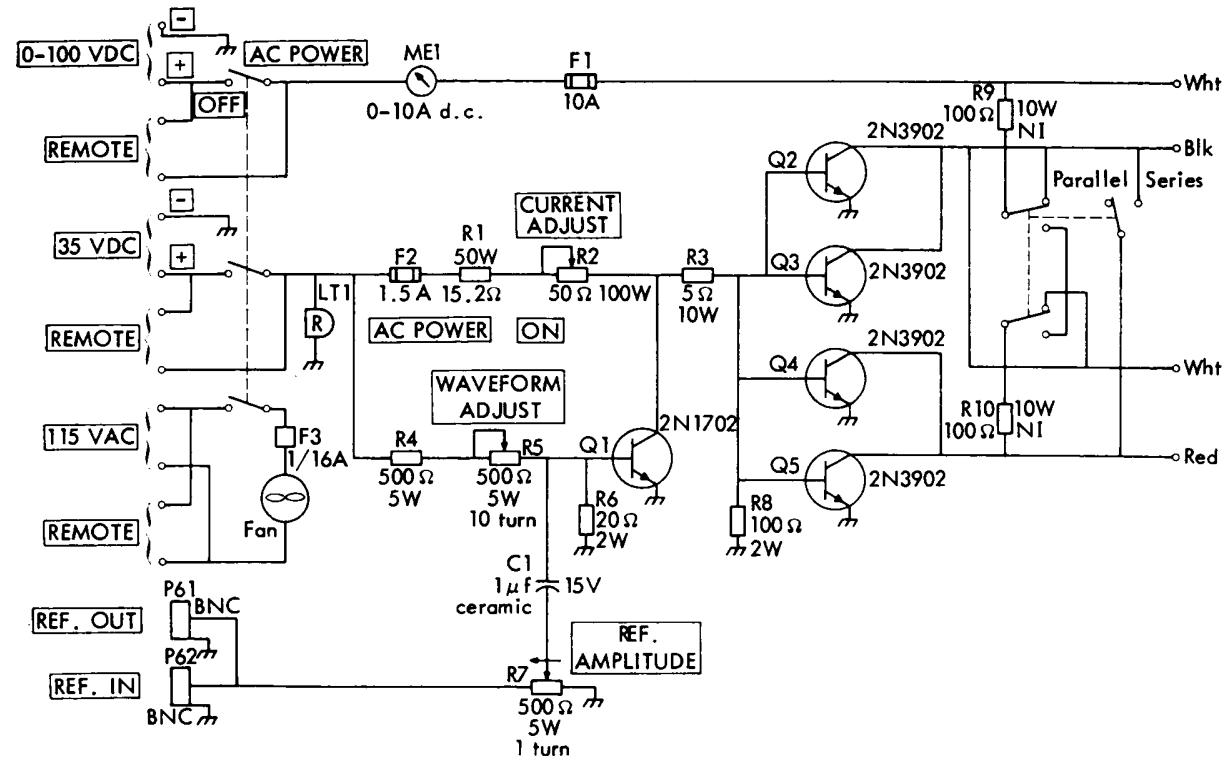

FIG. 2. Zeeman modulator (current switch) schematic diagram (all transistors are provided with heat sinks). crystal demodulates the carrier and the $10 \mathrm{kHz}$ signal is conducted to the preamplifier of the lock-in amplifier. The dc error signal derived by the lock-in amplifier is applied to the helix of the BWO, thus changing its output frequency. The polarity of the error signal is such that the frequency difference between the BWO and the cavity resonance is kept near zero. A change in the resonant frequency of the cavity then automatically causes the "slaved" BWO frequency to follow.

The experimentally measured frequency stability of our system, with a cavity of $Q_{L}=20000$, is 1 part in $10^{7} / \mathrm{sec}$ and 1 part in $10^{5} / \mathrm{h}$ in an air-conditioned room kept at $23^{\circ} \mathrm{C}$. With this kind of stability it becomes readily possible to reduce the frequency sweep rates to allow less than $1 \mathrm{~Hz}$ bandwidths for phase sensitive detection of molecular absorption.

\section{MODULATION AND DETECTION TECHNIQUE}

The modulation technique most commonly used in microwave rotational spectroscopy is called sample modulation. Here, one makes use of the interaction of an electric or magnetic field with a rotating molecule which causes a splitting in its energy levels. For the case of electric field interaction, which is most commonly used with molecules having permanent electric dipole moments, this splitting is called the Stark effect. When a molecule has a residual electronic spin angular momentum by virtue of its being paramagnetic, or by being in an electronic triplet state, a magnetic field can easily couple to the magnetic dipole, causing energy level splitting called the Zeeman effect. In either case, amplitude modulation of the gas absorption signal results from the application of an external field which is turned on and off at an appropriate rate. When the field is on, the intensity of the unsplit absorption line decreases due to the splitting caused by the field, and when it is off, the unsplit line is at full intensity. With this technique, ac amplification and phase sensitive detection techniques become possible (see next section).

The free radicals for which the spectrometer described in this paper was specifically designed are all paramagnetic by definition. That is, they all possess at least one unpaired electron spin that gives the molecule a resultant magnetic (Zeeman) modulation.

The use of Zeeman modulation in the study of free radicals becomes more profound when we consider the difficulties involved in searching for unknown spectra. Since most radicals exist for only a short time, there are usually other, more stable diamagnetic molecular species coexisting with the free radicals within the absorption cell. The stable molecules are likely to have rotational spectra in the same frequency region as the free radical. However, a system with Zeeman modulation is sensitive only to the paramagnetic species. One is not confronted, then, with the burdensome task of separating unwanted spectra.

The modulating magnetic field can be produced quite easily in the cavity spectrometer system by using either a pair of Helmholtz coils near the cavity, or a solenoid type coil wrapped around the cavity vacuum enclosure. So that the Zeeman lines ( $\sigma$ components) do not smear out the unsplit line, it is desirable to produce a magnetic field which is zero based and which turns on and off in the fastest possible time. The current through the coil windings should closely approximate a rectangular waveform. Because of the inherent inductance in the coil, the maximum frequency for which the current waveform approximates a rectangular shape is generally less than $10 \mathrm{kHz}$. A high power current switch, designed to produce the desired field, is shown schematically in Fig. 2. The circuit is a simple two-stage current switch with a single transistor (2N1702) driving four parallel, high voltage power transis- 
tors (2N3902). The high voltage capability is needed to prevent breakdown from the high back emf produced when the current through the inductance is shut off. Peak-to-peak currents of $10 \mathrm{~A}$ or more can be driven by this circuit.

Phase sensitive (or lock-in) detection is used in this spectrometer because of its distinct advantage over other types of homodyne detection schemes. By allowing extremely small bandwidth selectivity while at the same time effectively raising the frequency for amplification, phase sensitive detection and amplification minimize the common noise effects.

With reference to Fig. 1, the modulation and detection of a gas absorption occur in the following manner. The power reflected from the resonant cavity at its resonant frequency changes when a gas within the cavity absorbs at the same frequency. A rectangular current waveform supplied to the coils produces a magnetic field of nearly the same waveform inside the cavity. This field interacts with the molecules to split their rotational energy levels. The splitting effectively removes the gas absorption to other frequencies, causing an amplitude modulation of the signal reaching the detector crystal; this modulation is at the same frequency as that at which the Zeeman field is being switched. The output of the crystal detector (after demodulation of the carrier frequency) then has a frequency component identical with the frequency driving the Zeeman modulator. A reference frequency oscillator is used both to drive the Zeeman modulator (current switch) and as the reference for the phase detection. Since the frequency of the signal from the detector crystal is identical with the reference frequency, the lock-in amplifier produces a dc output proportional to the amplitude difference between them. A voltage vs time trace of the dc signal from the amplifier can then be observed on a chart recorder.

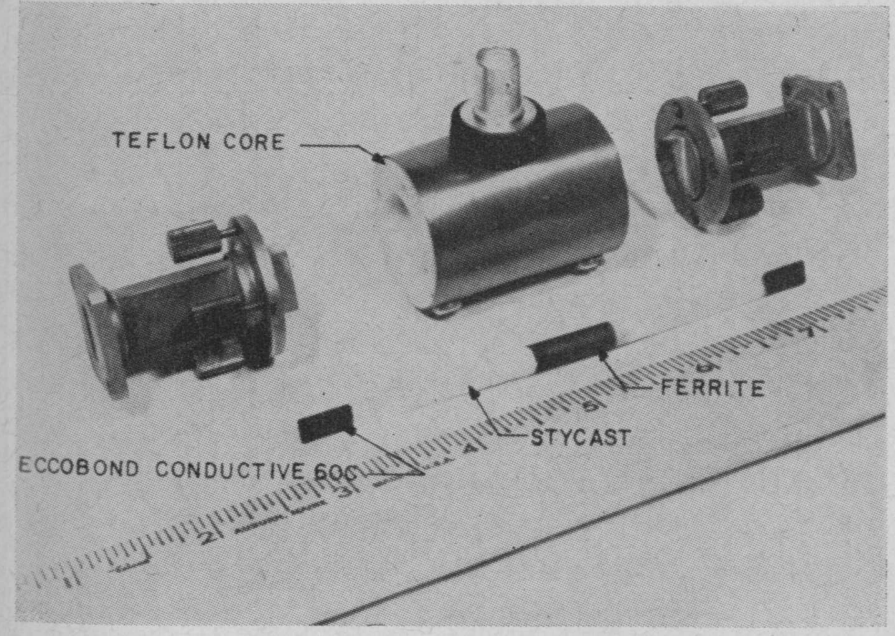

FIG. 3. Ferrite variable attenuator.

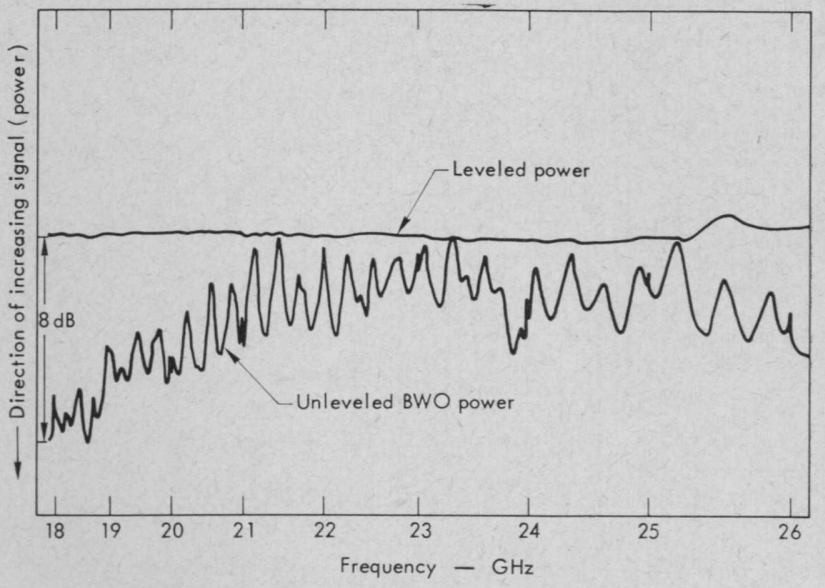

FIG. 4. BWO power leveled with ferrite attenuator.

\section{POWER LEVELING LOOP}

Standard microwave techniques can be used to assure a reasonably constant microwave power level entering the cavity absorption cell as the frequency changes. The desirability of a constant power level is conditioned by the need to determine accurate relative intensities of molecular absorptions within the cavity cell.

Two common methods are available to level microwave power from a BWO. One is to control the power generated by the oscillator by applying a signal to its control grid. This method has the disadvantage of causing unwanted frequency variations due to "pulling" effects, and the inconvenience of needing high voltage isolation. The other method is to attenuate the microwave power after it leaves the generator, with some kind of continuously variable device. No commercial device operable over the entire $\mathrm{K}$ band frequency range is known to be presently available, although at least one "homemade" device has been reported to operate successfully over this region. ${ }^{8}$

The need for an extremely broad banded variable device prompted the design of the ferrite variable attenuator shown in Fig. 3 and described in detail elsewhere. ${ }^{9} \mathrm{~A}$ ferrite rod has magnetic properties which can cause Faraday rotation of the microwave electromagnetic fields propagating on its surface. It is known that the degree of the rotation can be changed by magnetizing the ferrite rod by application of a low intensity field applied along its axis. Additionally, under certain conditions, such a rod acts as a "dielectric waveguide"; that is, it supports the propagation of microwave fields along its surface with low attenuation. Dielectric waveguides have notably large bandwidths. ${ }^{10}$ The device shown in Fig. 3 was used successfully in the spectrometer system to level power over all, or part, of the frequency region included by the system (see Fig. 4).

With reference again to Fig. 1, the leveling loop operates in the following manner. A portion $(-10 \mathrm{~dB})$ of the microwave power from the $\mathrm{BWO}$ is coupled from the main 


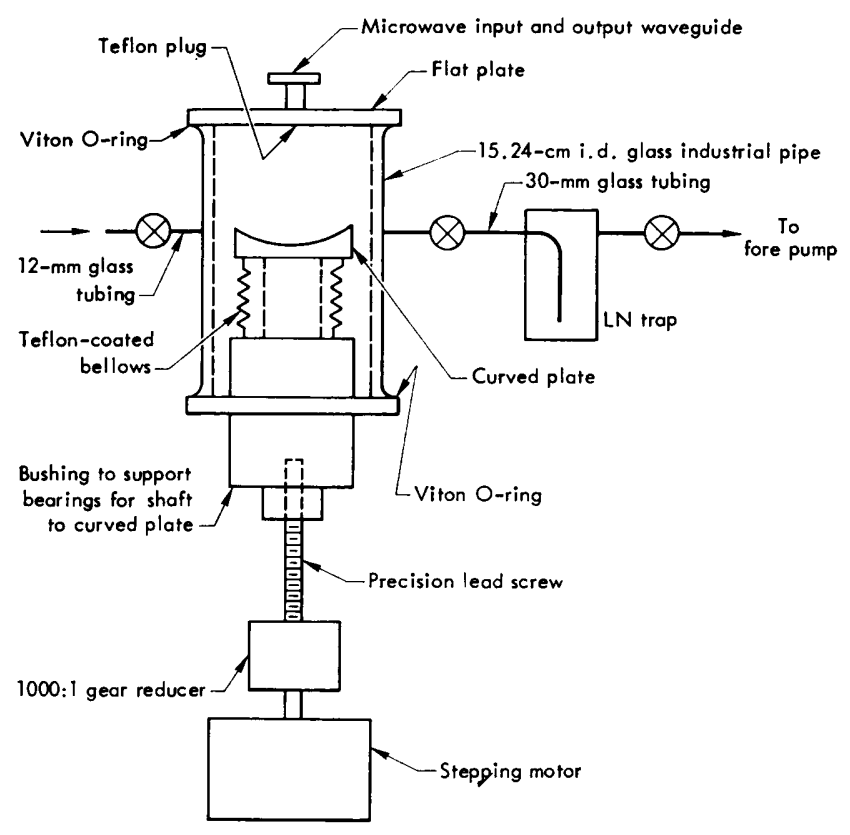

FIG. 5. Cavity spectrometer vacuum system.

transmission line and made incident on a thermistor detector. The detector responds to fluctuations in the incident power, causing a bridge in the power meter to unbalance. The amplified voltage signal from the power meter is then translated to a current to drive the solenoid coil on the ferrite rod. The field produced by the coil causes a rotation of the microwave fields traveling on the ferrite rod. An absorptive material is located strategically along the guide to absorb a portion of the incident energy. When initially biased to pass the lowest power level in a given frequency range, this loop can respond to up to $3 \mathrm{kHz}$ frequency variations and hold the power level to $\pm 0.3 \mathrm{~dB}$.

\section{FREQUENCY MEASUREMENT}

Precise frequency measurements of the spectra observed by a microwave rotational absorption spectrometer lead to accurate determinations of molecular parameters. Hence, it is desirable to determine the microwave generator frequency of the spectrometer in a simple but accurate manner.

A standard technique is used to count the $\mathrm{K}$ band frequencies (18-26.5 GHz) directly over the entire range. An electronically tunable, variable frequency oscillator (VFO) (180-270 $\mathrm{MHz}$ ) drives a step-recovery diode harmonic generator. The hundredth harmonic of the VFO frequency is mixed with the microwave frequency, and the VFO is tuned until their difference is some known frequency, say $30 \mathrm{MHz}$. The $30 \mathrm{MHz}$ intermediate frequency (i.f.) from the mixer is then phase compared with some other stable $30 \mathrm{MHz}$ reference (e.g., the crystal standard for the frequency counter) to derive an error signal used to tune the VFO. The VFO is then "phase locked" to the frequency of the microwave generator and follows any changes in the microwave frequency. When locked in this manner, the VFO frequency can be counted directly with a frequency counter. After we account for the $30 \mathrm{MHz}$ i.f. frequency difference, the VFO frequency is exactly $1 / 100$ of the microwave frequency, which then can be read to the accuracy of the counted VFO frequency.

To measure the frequency of a detected gas absorption signal, a marker can be derived from the frequency counter BCD output and displayed on the strip chart simultaneous with the recorder signal trace. The ability of the cavity to sweep bidirectionally over any part of the spectrum, coupled with its excellent short term stability, allows frequency determinations to $\pm 50 \mathrm{kHz}$ at $\mathrm{K}$ band frequencies.

The $30 \mathrm{MHz}$ reference frequency for the above system is derived from a $10 \mathrm{MHz}$ crystal (in the frequency counter) whose long term frequency stability is determined by comparing its phase with that of a standard signal broadcast by WWV at the National Bureau of Standards, Boulder, Colorado. A commercial instrument (HP 117 VLF comparator) was used to determine the stability of our standard (about 1 part in 108/day).

\section{THE RESONATOR AND DRIVE SYSTEM}

The semiconfocal Fabry-Perot type resonator used as the absorption cell consists of two aluminum plates. One plate is $17.78 \mathrm{~cm}$ in diameter and machined flat with a highly polished surface. The curved plate is $14.92 \mathrm{~cm}$ in diameter with a concave spherical surface (highly polished) of $30.48 \mathrm{~cm}$ radius of curvature. The resonator is operated with a plate separation close to $15.24 \mathrm{~cm}$ to ensure operation at the lowest loss resonances. The flat plate remains stationary while the curved plate is moved, to facilitate changing the cavity resonance to sweep the search frequency.

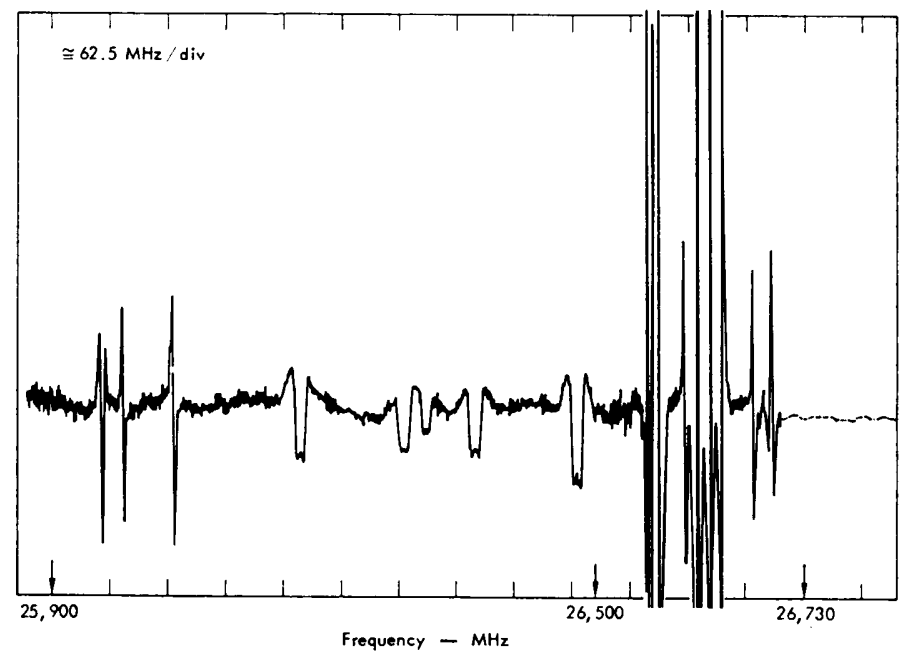

FIG. 6. $\mathrm{NO}_{2} \mathrm{~K}$-band rotational spectrum. 


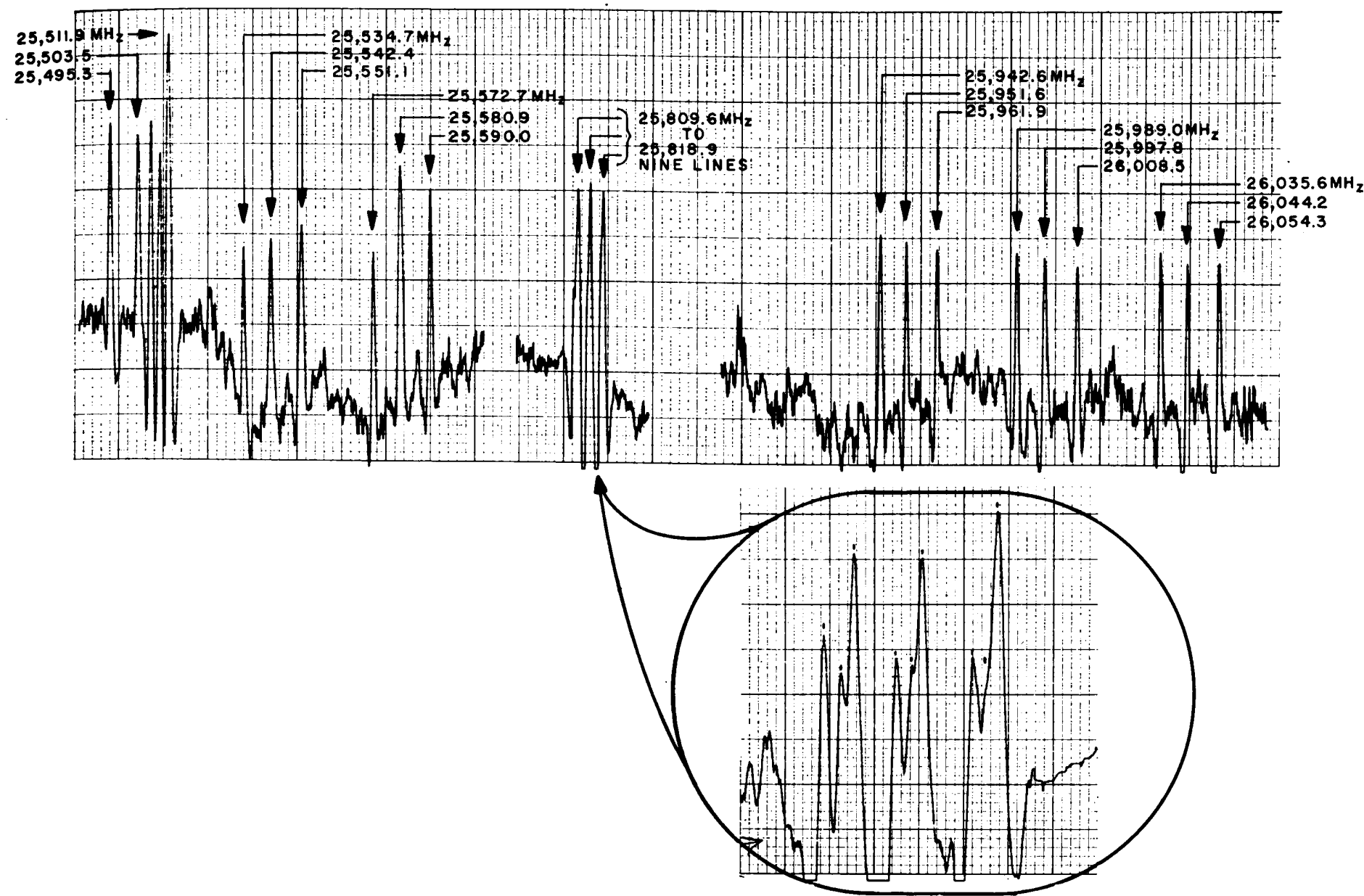

FIG. 7. $\mathrm{NF}_{2} \mathrm{~K}$-band rotational spectrum.

The curved plate is supported inside the vacuum system on a precision ground steel shaft (see Fig. 5). The shaft is supported by a large bearing and is driven vertically by a precision lead screw. A variable speed stepping motor drives the lead screw after a 1000:1 rotary speed reduction. This system operates over a range of sweep rates from $5 \mathrm{kHz} / \mathrm{sec}$ minimum to $10 \mathrm{MHz} / \mathrm{sec}$ maximum.

\section{VACUUM SYSTEM}

The vacuum system of the cavity spectrometer is quite simple. With reference to Fig. 5, the absorption cell consists of a Pyrex glass industrial pipe, $15.24 \mathrm{~cm}$ i.d. by $30.48 \mathrm{~cm}$ long, with O-ring grooved flanges on each end. Glass tubing outlets, located radially around the pipe, allow access to the absorption cell. The flat plate of the Fabry-Perot type cavity covers one end of the pipe, and the other end is sealed by a flange that supports the movable curved plate. The metal plates of the resonator and metal bellows inside the vacuum enclosure are covered with a thin layer of Teflon to reduce reactions with the highly reactive free radical species. A Teflon plug, 0.076 $\mathrm{mm}$ thick, seals the coupling hole of the waveguide entrance in the flat plate. A thermocouple gauge was used to measure the 100-200 mTorr pressures in the absorption cell. The remainder of the vacuum system consists of a large liquid nitrogen trap and a mechanical forepump.

\section{OBSERVED SPECTRA}

The successful location of previously unreported spectral lines of the $\mathrm{NO}_{2}$ and $\mathrm{NF}_{2}$ free radicals has verified the utility of the cavity system as a broad band search instrument.

Intensive work has been done by Bird et al. ${ }^{11}$ on the microwave spectrum of the $\mathrm{NO}_{2}$ molecule. In their statistical analysis, however, they were unable to use several lines observed in the $\mathrm{K}$ band because they had not observed the other lines belonging to the transition. Figure 6 shows what we believe to be the entire transition referred to above. All lines below $26480 \mathrm{MHz}$ have previously been unreported. This figure exhibits the system's capability of large continuous frequency sweeps while maintaining high sensitivity.

The real test of the usefulness of a search spectrometer would be the location of an unobserved spectrum. This is especially true for free radical searches where the experimental uncertainties can lead to many frustrating attempts. The cavity system has been used successfully to locate the $\mathrm{K}$ band rotational spectrum of the $\mathrm{NF}_{2}$ free 
radical. ${ }^{12}$ Structural information from infrared ${ }^{13}$ and electron diffraction ${ }^{14}$ studies allowed the determination of possible frequency regions in which to make intensive searches. Figure 7 shows a group of hyperfine lines for two rotational transitions of the $\mathrm{NF}_{2}$ free radical. Each rotational transition is split by the interaction of the unpaired electron-spin angular momentum, with the molecular rotation and each of the nuclear spins within the molecule, in the same manner as for $\mathrm{NO}_{2}{ }^{11}$ and $\mathrm{ClO}_{2} \cdot{ }^{15} \mathrm{~A}$ total of 18 hyperfine lines is expected for each $\mathrm{NF}_{2}$ transition. An example of the resolution facilitated by the slow sweep capabilities of the system is shown by the inset in Fig. 7 .

The cavity spectrometer system is easily adapted to gas flow studies for free radicals or excited species produced in glow discharges. A microwave discharge cavity can be positioned arbitrarily close to the absorption cell to optimize the radical concentration, and the high volumeto-surface ratio of the open Fabry-Perot type cavity limits radical recombination by surface collisions. $\mathrm{K}$ band absorption lines for the $\mathrm{OH}$ free radical have been observed with this system in the gas flow products from a microwave discharge through water vapor.

Stark modulation can be produced quite easily with this system by using the two plates of the cavity as electrodes. The $J=16, K=14 \mathrm{NH}_{3}$ inversion absorption line at $24777 \mathrm{MHz}$ (intensity approximately $1.8 \times 10^{-7} \mathrm{~cm}^{-1}$ ) was obtained with Stark modulation on this system with a signal-to-noise of about 30:1. It was observed, however, that several disadvantages limit the usefulness of Stark modulation on this type of system. Because of the geometry of the plates, the field gradient is not uniform between them and consequently, the locally high field gradients within the cavity can cause ionization of the sample gas at the higher peak-to-peak voltage levels. Also, the plate separation and therefore the field gradient change during the sweep.

The microwave cavity spectrometer described in this paper has been shown to be very sensitive to weak molecu- lar absorptions, to have inherent frequency stability, to have utility as a broad band search spectrometer, to be readily adaptable to free radical studies, and to be versatile in its operation. The extension of the design principles for this $\mathrm{K}$ band system to high frequency regions (even into the submillimeter region) is direct and is our next consideration.

\section{ACKNOWLEDGMENTS}

We would like to thank technicians Robert S. Hodge, Bruce H. Shue, and James M. Spann for their exceptional support during this project.

\footnotetext{
* Work performed under the auspices of the U. S. Atomic Energy Commission.

$\dagger$ Work done as part of doctoral dissertation for the University of Wyoming, Laramie, Wyoming.

$\ddagger$ University of Wyoming, Laramie, Wyoming.

${ }^{1}$ G. C. Dousmanis, T. M. Sanders, Jr., and C. H. Townes, Phys. Rev. 100, 1735 (1955).

${ }^{2}$ F. X. Powell and D. R. Lide, J. Chem. Phys. 41, 1413 (1964).

${ }^{3} \mathrm{H}$. E. Radford and M. Linzer, Phys. Rev. Lett. 10, 443 (1963).

${ }^{4} \mathrm{D}$. R. Johnson, "The Zeeman Effect in the Microwave Spectra of Free Radicals," unpublished doctoral dissertation, Univ. Okla., 1967.

${ }^{5}$ W. H. Harrington, J. Chem. Phys. 46, 3698 (1967).

${ }^{6}$ L. H. Hrubesh, R. E. Anderson, and E. A. Rinehart, "A Theoretical Discussion of a K-band Microwave Cavity Resonator Spectrometer," Lawrence Radiation Laboratory, Livermore, Rept. UCRL50739, 1969.

${ }^{7}$ L. W. Hrubesh, "Free Radical Microwave Rotational Spectroscopy with a Resonant Cavity" [doctoral dissertation, Univ. Wyo. (1970) ], Lawrence Radiation Laboratory, Livermore, Rept. UCRL50890, 1970, Appendix A.

${ }^{8}$ E. A. Rinehart and R. L. Legan, Rev. Sci. Instrum. 35, 103 (1964)

${ }^{9}$ L. W. Hrubesh, Microwave J. 13, 75 (1970).

${ }^{10}$ C. H. Chandler, J. Appl. Phys. 20, 1188 (1949).

${ }_{11}$ G. R. Bird, J. C. Baird, A. W. Jache, J. A. Hodgeson, R. F. Curl, A. W. Kunkle, J. W. Bransford, J. R. Anderson, and J. Rosenthal, J. Chem. Phys. 40, 3378 (1964).

${ }_{12}$ L. W. Hrubesh, E. A. Rinehart, and R. E. Anderson, J. Mol. Spectrosc. 36, 354 (1970).

${ }_{13}$ M. D. Harmony, R. J. Myers, L. J. Schoen, D. R. Lide, and D. E. Mann, J. Chem. Phys. 35, 1129 (1961).

${ }^{14}$ R. K. Bohn and S. H. Bauer, Inorg. Chem. 6, 304 (1967).

${ }_{15}$ R. F. Curl, R. F. Heidelberg, and J. L. Kinsey, Phys. Rev. 121, 1119 (1961).
} 